\title{
CIRCULATION OF CAR TRAFFIC IN CONGESTED URBAN AREAS*
}

\author{
ANNUNZIATA CASCONE ${ }^{\dagger}, \mathrm{CIRO}^{\prime} \mathrm{DPICE}^{\ddagger}, \mathrm{BENEDETTO}$ PICCOLI $^{\S}$, AND LUIGI \\ RARITÀ
}

\begin{abstract}
The aim of this work is to understand how urban traffic behavior, especially in cases of congestion, can be improved by an accurate choice of traffic coefficients. For this, we define three cost functionals that measure average velocity, average travelling time and total flux of cars. The global optimal control problem for a complex network is difficult to solve both from analytical and numerical points of view. Thus, we focus on a simple junction with one incoming road and two outgoing roads (junctions of $1 \times 2$ type), obtaining exact solutions to a simple optimization problem. Then, we use such results at each node of the network. The traffic evolution of some networks is then studied via simulations. In particular, it is shown that an appropriate choice of the traffic distributions can be useful in order to improve network conditions.
\end{abstract}

Key words. Traffic flows on road networks, conservation laws, optimal control

AMS subject classifications. 35L65, 49J20, 76N25

\section{Introduction}

This paper is devoted to problems of car traffic flows on urban networks in the case where congestion occurs. A fluid dynamic model for road networks, based on traffic distribution parameters, is considered. Then, redirection of cars through an opportune choice of distribution parameters is studied in the case of critical car densities.

We focus on the recent approach for networks based on conservation laws ([5, $6,9,15,16])$. Some optimization problems for road networks have been already studied; [13] is devoted to traffic light regulation, while [12] and [14] are more related to our analysis but focus on the case of smooth solutions (not developing shocks) and boundary control. The same approach was used in [3], but the optimization refers to right of way parameters for networks with a number of incoming roads greater than the number of outgoing ones.

Junctions of a network are fundamental, as the system at a junction is underdetermined even after imposing the conservation of cars. In order to uniquely solve the Riemann problem at junctions (the problem with initial data constant on each road), we make the assumption (see [6]) that the incoming traffic distributes to outgoing roads according to fixed (statistical) distribution coefficients, and that drivers behave in order to maximize the through flux.

Our aim is to optimize the performance of congested road networks through an accurate choice of the distribution coefficients. In reality, such coefficients are fixed by drivers habits; however, drivers preferences can change due to some strong phenomena on roads, such as congestion or intervention by local police.

${ }^{*}$ Received: December 20, 2007; accepted (in revised version): June 11, 2008. Communicated by Lorenzo Pareschi.

${ }^{\dagger}$ Department of Information Engineering and Applied Mathematics, University of Salerno, Fisciano (SA), Italy (cascone@diima.unisa.it).

$\ddagger$ Department of Information Engineering and Applied Mathematics, University of Salerno, Fisciano (SA), Italy (dapice@diima.unisa.it).

§Istituto per le Applicazioni del Calcolo "Mauro Picone", Consiglio Nazionale delle Ricerche, Roma, Italy (b.piccoli@iac.cnr.it).

IDepartment of Information Engineering and Applied Mathematics, University of Salerno, Fisciano (SA), Italy (lrarita@unisa.it). 
In order to give some optimization criteria for road networks and, in particular, for the study of the distribution coefficients, three cost functionals are considered. The cost functionals, called, respectively, $J_{1}, J_{2}$, and $J_{3}$, measure average velocity, average travelling time, and flux of cars.

The same functionals were considered in [3] and [4]. However, [3] focuses on yielding rules, thus considers the case of junctions with two incoming and one outgoing road, while [4] is based on optimization of traffic distribution coefficients for a telecommunication network model with $2 \times 2$ junctions (two incoming lines and two outgoing lines). In particular, the telecommunication networks model for $2 \times 2$ junctions is different from the vehicular traffic one used here. This is reflected in a quite different mathematical problem. The analysis of the performance of the network through the functionals $J_{1}, J_{2}$, and $J_{3}$ is a very delicate problem, since a complete mathematical study of a complex network is very difficult, especially in case of critical conditions, such as congestion.

For this reason, an exact solution is found for single junctions and asymptotic costs. The global (sub)optimal control for more complex networks is obtained by localization: the exact optimal solution is applied locally for every junction of the network and at every time. Notice that this approach provides only preliminary results, in fact only a suboptimal solution is given. However, it has the advantage of giving explicit controls and allowing a decentralized policy.

In detail, the attention is focused on a simple junction, that consists of one incoming and two outgoing roads $(1 \times 2)$. This type of junction needs only one traffic distribution coefficient $\alpha$. The optimal value of $\alpha$ is found for cost functionals $J_{1}$ and $J_{2}$. For $J_{3}$, the optimal choice of $\alpha$ was already illustrated in [3].

The optimization algorithm is then applied to one network with junctions of $1 \times 2$ type. For numerics, we refer to approximation methods of $[2,10]$ and [11]. Simulations are carried out with three different cases for the choice of the distribution coefficients: (locally) optimal, fixed, and dynamic random. The first choice is represented by the optimization algorithm and the second one is obtained by considering a fixed value for the distribution coefficients of every junction. For dynamic random simulations the distribution coefficients are randomly chosen at every instant of time and for every junction independently. The results of such approach are very similar to those obtained with the optimization algorithm. In order to make a distinction among the two approaches, the Stop and Go Waves functional (briefly $S G W$ ), is considered (see [3] and [7]). The $S G W$ functional is a performance parameter that measures the velocity variation, one of the main causes of car accidents. This functional is very high for the dynamic random case and very low for the optimal one. Moreover, in order to establish the phenomena connected with a dynamic random approach, we refer to the theory of stochastic processes and their ergodicity (see [18]). It is simple to show that the dynamic random algorithm behaves similarly to a particular fixed case, with all distribution coefficients equal to 0.5 .

The paper is organized as follows. In section 2, we recall the construction of solutions to Riemann problems at junctions, using the approach of [5] and [6]. Section 3 is devoted to the optimization, introducing the three functionals $J_{i}, i=1, \ldots, 3$. Then, section 4 reports simulation results for the three different choices of parameters: optimal, fixed, and dynamic random. We discuss results and use another functional, the Stop and Go Waves one, to distinguish among the optimal and dynamic random case. A statistical analysis of the dynamic random simulations is also presented. The paper ends with conclusions in section 5 . 


\section{Riemann solver for the road network}

In this section, we shortly describe a fluid dynamic model for road networks. For further details, see [6], and [8].

A road network is given by a couple $(\mathcal{I}, \mathcal{J})$, where $\mathcal{I}=\left\{I_{i}=\left[a_{i}, b_{i}\right] \subseteq \mathbb{R}, i=1, \ldots, N\right\}$ represents a finite set of edges (roads), and $\mathcal{J}$ is the collection of vertices. Each vertex $J$ is union of two nonempty subsets $\operatorname{Inc}(J)$ and $\operatorname{Out}(J)$ of $\{1, \ldots, N\}$ representing, respectively, the incoming and the outgoing roads. We assume that for every $J \neq J^{\prime} \in$ $\mathcal{J}, \operatorname{Inc}(J) \cap \operatorname{Inc}\left(J^{\prime}\right)=\varnothing$ and $\operatorname{Out}(J) \cap \operatorname{Out}\left(J^{\prime}\right)=\varnothing$. This means that each edge can be incoming for at most one vertex and outgoing for at most one vertex.

On each road, we consider the Lighthill-Whitham-Richards model, described by the equation (see [17], [19])

$$
\partial_{t} \rho+\partial_{x} f(\rho)=0,
$$

where $\rho=\rho(t, x) \in\left[0, \rho_{\max }\right]$ is the density of cars, $\rho_{\max }$ is the maximal density of cars, $f(\rho)=\rho v(\rho)$ is the flux and $v(\rho)$ is the average velocity. For roads such that $i \notin$ $\cup_{J \in \mathcal{J}} \operatorname{Inc}(J)$ and $b_{i}<+\infty$ or such that $i \notin \cup_{J \in \mathcal{J}}$ Out $(J)$ and $a_{i}>-\infty$, a boundary condition is needed.

We assume that:

(H) $f$ is a strictly concave $C^{2}$ function, and that $f(0)=f\left(\rho_{\max }\right)=0$.

Setting $\rho_{\max }=1$ and $v(\rho)=1-\rho$, one example of a flux function ensuring $(\mathrm{H})$ is the following:

$$
f(\rho)=\rho(1-\rho), \rho \in[0,1],
$$

which has a unique maximum $\sigma=\frac{1}{2}$. This flux function satisfies all the previous assumptions (H). In fact, $f(0)=f(1)=0$, and $f^{\prime \prime}(\rho)=-2<0$.

For future use, we define

Definition 2.1. Let $\tau:[0,1] \rightarrow[0,1]$ be the map such that $f(\tau(\rho))=f(\rho)$ for every $\rho \in[0,1]$ and $\tau(\rho) \neq \rho$ for every $\rho \in[0,1] \backslash\left\{\frac{1}{2}\right\}$.

For a single conservation law (2.1) on a real line $\mathbb{R}$, a Riemann problem (RP) is a Cauchy problem for an initial data which is piecewise constant with only one discontinuity. In a similar way, we define

Definition 2.2. A Riemann Problem (RP) at a junction is a Cauchy problem for an initial data constant on each road.

We now describe a systematic way of solving RPs at junctions. Fix a junction $J$ with $n$ incoming roads and $m$ outgoing ones, where $I_{i}, i=1, \ldots, n$, are the incoming roads and $I_{j}, j=n+1, \ldots, n+m$, are the outgoing ones. Let $\rho=\left(\rho_{1}, \ldots, \rho_{n+m}\right), \rho_{i} \in$ $[0,+\infty] \times I_{i}$ be the density vector for $J$.

Definition 2.3. A Riemann solver for the junction $J$ is a map $R S:[0,1]^{n} \times[0,1]^{m} \rightarrow$ $[0,1]^{n} \times[0,1]^{m}$ that associates to Riemann data $\rho_{0}=\left(\rho_{1,0}, \ldots, \rho_{n+m, 0}\right)$ at $J$ a vector $\widehat{\rho}=\left(\widehat{\rho}_{1}, \ldots, \widehat{\rho}_{n+m}\right)$ so that the solution on an incoming road $I_{i}, i=1, \ldots, n$, is given by the wave $\left(\rho_{i, 0}, \widehat{\rho}_{i}\right)$, and on an outgoing road $I_{j}, j=n+1, \ldots, n+m$, is given by the wave $\left(\widehat{\rho}_{j}, \rho_{j, 0}\right)$. We require the consistency condition

$$
\text { (CC) } R S\left(R S\left(\rho_{0}\right)\right)=R S\left(\rho_{0}\right) .
$$

If $m \geq n$, it is possible to introduce a Riemann solver, based on the following rules $([6,8])$ : 
(A) At each junction $J$, we define a matrix $A=\left(\alpha_{j, i}\right)$, that describes the traffic distribution from incoming to outgoing roads, where, for every $i \in\{1, . ., n\}$ and $j \in\{n+1, \ldots, n+m\}, 0 \leq \alpha_{j, i} \leq 1$ and $\sum_{j=n+1}^{n+m} \alpha_{j, i}=1$.

The $i$-th column of $A$ indicates the percentages of traffic that, from the incoming road $I_{i}$, distribute to outgoing roads.

(B) Respecting (A), drivers behave so as to maximize the flux through $J$.

Let us focus on a junction $J$, characterized by one incoming road, $a$, and $n$ outgoing roads, indicated by $b_{1}, b_{2}, . ., b_{n}$ (see Figure 2.1). We indicate the car density

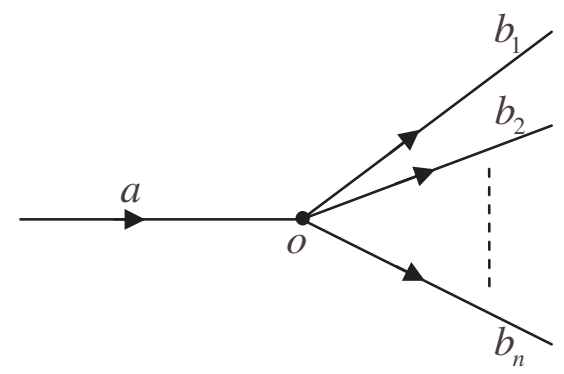

FIG. 2.1. Junction with one incoming road and $n$ outgoing roads.

for road $a$ by $\rho_{a}(t, x) \in[0,1],(t, x) \in \mathbb{R}^{+} \times I_{a}$, and for roads $b_{j}, j=1, \ldots, n$, by $\rho_{b_{j}}(t, x) \in$ $[0,1],(t, x) \in \mathbb{R}^{+} \times I_{b_{j}}$. The maximal flux values on roads are described as follows (for a proof see $[8])$ :

Proposition 2.4. For the flux function (2.2), let $\left(\rho_{a, 0}, \rho_{\psi, 0}\right), \psi=b_{j}, j=1, \ldots, n$, be the initial densities of a RP at $J$ and let $\gamma_{a}^{\max }$ and $\gamma_{\psi}^{\max }, \psi=b_{j}, j=1, \ldots, n$, be the maximum fluxes that can be obtained on the incoming road and the outgoing roads, respectively. Then

$$
\begin{gathered}
\gamma_{a}^{\max }= \begin{cases}f\left(\rho_{a, 0}\right), & \text { if } \rho_{a, 0} \in\left[0, \frac{1}{2}\right], \\
f\left(\frac{1}{2}\right), & \text { if } \left.\left.\rho_{a, 0} \in\right] \frac{1}{2}, 1\right],\end{cases} \\
\gamma_{\psi}^{\max }= \begin{cases}f\left(\frac{1}{2}\right), & \text { if } \rho_{\psi, 0} \in\left[0, \frac{1}{2}\right], \quad \psi=b_{j}, j=1, \ldots, n . \\
f\left(\rho_{\psi, 0}\right), & \text { if } \left.\left.\rho_{\psi, 0} \in\right] \frac{1}{2}, 1\right],\end{cases}
\end{gathered}
$$

The parameter $\left.\alpha_{i} \in\right] 0,1\left[, \sum_{i=1}^{n} \alpha_{i}=1\right.$, indicates the percentage of cars that, from road $a$, go to the outgoing road $b_{i}, i=1, \ldots, n$. The fluxes of the solution to the RP for $J$ are given by

$$
\widehat{\gamma}=\left(\widehat{\gamma}_{a}, \widehat{\gamma}_{b_{1}}, \ldots, \widehat{\gamma}_{b_{n}}\right)=\left(\widehat{\gamma}_{a}, \alpha_{1} \widehat{\gamma}_{a}, \ldots, \alpha_{n} \widehat{\gamma}_{a}\right)
$$

where

$$
\widehat{\gamma}_{a}=\min \left\{\gamma_{a}^{\max }, \frac{\gamma_{b_{1}}^{\max }}{\alpha_{1}}, \ldots, \frac{\gamma_{b_{n}}^{\max }}{\alpha_{n}}\right\}
$$

while the corresponding density solution $\widehat{\rho}$ is found as follows (see also [3], [6], and $[8])$ :

$$
\widehat{\rho}_{a} \in \begin{cases}\left.\left.\left\{\rho_{a, 0}\right\} \cup\right] \tau\left(\rho_{a, 0}\right), 1\right], & \text { if } 0 \leq \rho_{a, 0} \leq \frac{1}{2} \\ {\left[\frac{1}{2}, 1\right],} & \text { if } \frac{1}{2} \leq \rho_{a, 0} \leq 1\end{cases}
$$


and

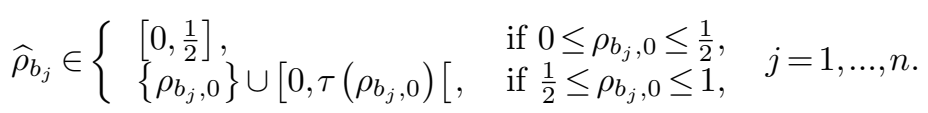

For the incoming road, the solution is given by the wave $\left(\rho_{a, 0}, \widehat{\rho}_{a}\right)$, while for $j=1, \ldots, n$, the solution is given by the wave $\left(\widehat{\rho}_{b_{j}}, \rho_{b_{j}, 0}\right)$. In [8], there are also existence results for the Cauchy problems.

In the following, we use the time-varying parameters $t \rightarrow \alpha_{i}(t)$ as controls for the optimization of traffic flows.

REMARK 2.5. Notice that the approach is equivalent to the supply-demand framework introduced by Lebacque. In fact, $\gamma_{a}^{\max }$ is the demand and $\gamma_{\psi}^{\max }$ the supply for the connected roads (see also [8], section 5.2.3, page 106).

\section{Cost functionals for single junctions}

Given an initial data $\left(\rho_{a, 0}, \rho_{b_{j}, 0}\right), j=1, . ., n$, for the network of Figure 2.1 we can define the following cost functionals, that indicate average velocity, average travelling time, and flux, respectively:

$$
\begin{aligned}
& J_{1}(t)=\int_{I_{a}} v\left(\rho_{a}(t, x)\right) d x+\sum_{j=1}^{n} \int_{I_{b_{j}}} v\left(\rho_{b_{j}}(t, x)\right) d x, \\
& J_{2}(t)=\int_{I_{a}} \frac{1}{v\left(\rho_{a}(t, x)\right)} d x+\sum_{j=1}^{n} \int_{I_{b_{j}}} \frac{1}{v\left(\rho_{b_{j}}(t, x)\right)} d x, \\
& J_{3}(t)=\int_{I_{a}} f\left(\rho_{a}(t, x)\right) d x+\sum_{j=1}^{n} \int_{I_{b_{j}}} f\left(\rho_{b_{j}}(t, x)\right) d x .
\end{aligned}
$$

For a fixed time horizon $[0, T]$ our aim is to maximize $\int_{0}^{T} J_{1}(t) d t$ and $\int_{0}^{T} J_{3}(t) d t$ and to minimize $\int_{0}^{T} J_{2}(t) d t$ by choosing the traffic distribution coefficient $\alpha_{i}(t)$. Since the solutions of such optimization control problems are very difficult, we consider the following problem:

(P) Consider a junction $J$ as in Figure 2.1, the traffic distribution coefficients $\alpha_{i}$ as controls, and the functionals $J_{i}, i=1,2,3$. We want to maximize $J_{1}(T)$ and $J_{3}(T)$, and minimize $J_{2}(T)$ for $T$ sufficiently large.

3.1. $n=1$ incoming road and $m=2$ outgoing roads. In what follows, we fix the flux function (2.2) and focus on the special case of a junction of type $1 \times 2$. We call $b_{1}=b$ and $b_{2}=c$ the two outgoing roads, respectively. The cost functionals depend on only one parameter $\alpha \in] 0,1$ [, which represents the percentage of drivers who, from road $a$, go to road $b$. For this type of network and for $T$ sufficiently large, the cost functionals $J_{1}(T), J_{2}(T)$ and $J_{3}(T)$ can be written as:

$$
\begin{aligned}
& J_{1}(T)=v\left(\widehat{\rho}_{a}\right)+v\left(\widehat{\rho}_{b}\right)+v\left(\widehat{\rho}_{c}\right), \\
& J_{2}(T)=\frac{1}{v\left(\widehat{\rho}_{a}\right)}+\frac{1}{v\left(\widehat{\rho}_{b}\right)}+\frac{1}{v\left(\widehat{\rho}_{c}\right)}, \\
& J_{3}(T)=f\left(\widehat{\rho}_{a}\right)+f\left(\widehat{\rho}_{b}\right)+f\left(\widehat{\rho}_{c}\right) .
\end{aligned}
$$


By direct computation:

$$
\begin{gathered}
J_{1}(T)=\frac{3}{2}-\frac{1}{2}\left[s_{a} \sqrt{1-4 \hat{\gamma}_{a}}+s_{b} \sqrt{1-4 \hat{\gamma}_{b}}+s_{c} \sqrt{1-4 \hat{\gamma}_{c}}\right], \\
J_{2}(T)=\frac{2}{1-s_{a} \sqrt{1-4 \hat{\gamma}_{a}}}+\frac{2}{1-s_{b} \sqrt{1-4 \hat{\gamma}_{b}}}+\frac{2}{1-s_{c} \sqrt{1-4 \hat{\gamma}_{c}}}, \\
J_{3}(T)=\widehat{\gamma}_{a}+\widehat{\gamma}_{b}+\widehat{\gamma}_{c},
\end{gathered}
$$

where $s_{a}$ and $s_{\psi}, \psi=b, c$, are defined as:

$$
\begin{gathered}
s_{a}= \begin{cases}+1 \quad \text { if } \rho_{a, 0} \geq \frac{1}{2}, \\
\quad \text { or } \rho_{a, 0}<\frac{1}{2} \text { and } \gamma_{a}^{\max }>\min \left\{\frac{\gamma_{b}^{\max }}{{ }^{\alpha}}, \frac{\gamma_{c}^{\max }}{1-\alpha}\right\}, \\
-1 \quad \text { if } \rho_{a, 0}<\frac{1}{2} \text { and } \gamma_{a}^{\max } \leq \min \left\{\frac{\gamma_{b}^{\max }}{\alpha}, \frac{\gamma_{c}^{\max }}{1-\alpha}\right\},\end{cases} \\
s_{\psi}= \begin{cases}+1 \quad \text { if } \rho_{\psi, 0}>\frac{1}{2} \text { and } \frac{\gamma_{\psi}^{\max }}{\alpha_{\psi}} \leq \min \left\{\gamma_{a}^{\max }, \frac{\gamma_{\psi^{\prime}}^{\max }}{\alpha_{\psi^{\prime}}}\left(\psi^{\prime} \neq \psi\right)\right\}, \\
-1 \quad \text { if } \rho_{\psi, 0} \leq \frac{1}{2}, \\
\text { or } \rho_{\psi, 0}>\frac{1}{2} \text { and } \frac{\gamma_{\psi}^{\max }}{\alpha_{\psi}}>\min \left\{\gamma_{a}^{\max }, \frac{\gamma_{\psi^{\prime}}^{\max }}{\alpha_{\psi^{\prime}}}\left(\psi^{\prime} \neq \psi\right)\right\},\end{cases}
\end{gathered}
$$

with

$$
\alpha_{\psi}= \begin{cases}\alpha, \quad \text { if } \psi=b \\ 1-\alpha, & \text { if } \psi=c\end{cases}
$$

If $\widehat{\gamma}_{a}=\gamma_{a}^{\max }$, then the solution to the RP is:

$$
\widehat{\gamma}=\left(\gamma_{a}^{\max }, \alpha \gamma_{a}^{\max },(1-\alpha) \gamma_{a}^{\max }\right) .
$$

We have to maximize

$$
\widehat{J}_{1}=-s_{b} \sqrt{1-4 \alpha \gamma_{a}^{\max }}-s_{c} \sqrt{1-4(1-\alpha) \gamma_{a}^{\max }},
$$

and minimize

$$
\widehat{J}_{2}=\frac{1}{1-s_{b} \sqrt{1-4 \alpha \gamma_{a}^{\max }}}+\frac{1}{1-s_{c} \sqrt{1-4(1-\alpha) \gamma_{a}^{\max }}},
$$

where $\widehat{J}_{1}$ and $\widehat{J}_{2}$ are the parts of (3.1) and (3.2) that depend on $\alpha$. On the other hand, $J_{3}=2 \gamma_{a}^{\max }$ is independent of $\alpha$.

If $\widehat{\gamma}_{a}=\frac{\gamma_{b}^{\max }}{\alpha}$ or $\widehat{\gamma}_{a}=\frac{\gamma_{c}^{\max }}{1-\alpha}$, then the solution to the RP can be written as:

$$
\widehat{\gamma}=\left(\widehat{\gamma}_{a, \psi}, \alpha \widehat{\gamma}_{a, \psi},(1-\alpha) \widehat{\gamma}_{a, \psi}\right) \text {, }
$$

where

$$
\widehat{\gamma}_{a, \psi}= \begin{cases}\frac{\gamma_{c}^{\max }}{1-\alpha}, & \text { if } \psi=b, \\ \frac{\gamma_{b}^{\max }}{\alpha} & \text { if } \psi=c .\end{cases}
$$

In this case, the expressions of $\widehat{J}_{1}$ and $\widehat{J}_{2}$ are:

$$
\widehat{J}_{1}=-s_{a} \sqrt{1-4 \widehat{\gamma}_{a, \psi}}-s_{b} \sqrt{1-4 \alpha \widehat{\gamma}_{a, \psi}}-s_{c} \sqrt{1-4(1-\alpha) \widehat{\gamma}_{a, \psi}},
$$

and

$$
\widehat{J}_{2}=\frac{1}{1-s_{a} \sqrt{1-4 \widehat{\gamma}_{a, \psi}}}+\frac{1}{1-s_{b} \sqrt{1-4 \alpha \hat{\gamma}_{a, \psi}}} \cdot+\frac{1}{1-s_{c} \sqrt{1-4(1-\alpha) \widehat{\gamma}_{a, \psi}}} .
$$

It can be verified that $J_{3}$ is constantly equal to $2 \widehat{\gamma}_{a, \psi}$. 
3.1.1. Optimal choice of the distribution coefficient $\alpha$. First, we give a result for functionals, which are monotone with respect to the flux, in particular including the case of $J_{3}$.

Proposition 3.1. Let $m:\left[0, f_{\max }\right] \rightarrow \mathbb{R}$ be a monotone function, and define

$$
M(t)=\int_{I_{a}} m\left(f\left(\rho_{a}(t, x)\right)\right) d \rho_{a}+\sum_{j=1}^{n} \int_{I_{b_{j}}} m\left(f\left(\rho_{b_{j}}(t, x)\right)\right) d \rho_{b_{j}},
$$

and

$$
\bar{\alpha}=\frac{\gamma_{b}^{\max }}{\gamma_{b}^{\max }+\gamma_{c}^{\max }} .
$$

If

$$
\gamma_{a}^{\max } \leq \min \left\{\frac{\gamma_{b}^{\max }}{\bar{\alpha}}, \frac{\gamma_{c}^{\max }}{1-\bar{\alpha}}\right\}
$$

then the maximal value of $M(T)$ for $T$ sufficiently large, is obtained for all the values of $\alpha$ such that (3.4) holds true (with $\bar{\alpha}$ replaced by $\alpha$ ). Otherwise, $M(T)$ is maximized only for $\alpha=\bar{\alpha}$.

Proof. The value of $\bar{\alpha}$ is such that

$$
\frac{\gamma_{b}^{\max }}{\bar{\alpha}}=\frac{\gamma_{c}^{\max }}{1-\bar{\alpha}} .
$$

Assume first that (3.4) holds true. For $\alpha$ verifying (3.4), the solution to the RP is given by $\widehat{\gamma}=\left(\gamma_{a}^{\max }, \alpha \gamma_{a}^{\max },(1-\alpha) \gamma_{a}^{\max }\right)$ and $M(T)=2 \gamma_{a}^{\max }$. For the other values, $\gamma_{a}^{\max }>\widehat{\gamma}_{a, \psi}$ and $M(T)=2 \widehat{\gamma}_{a, \psi}$. Then, the conclusion follows.

Assume now that (3.4) is false. Then, the solution to the RP is always

$$
\widehat{\gamma}=\left(\widehat{\gamma}_{a, \psi}, \alpha \widehat{\gamma}_{a, \psi},(1-\alpha) \widehat{\gamma}_{a, \psi}\right),
$$

where $\psi=b$ for $\alpha \leq \bar{\alpha}$, while $\psi=c$ for $\alpha \geq \bar{\alpha}$. If $\psi=b$, the maximum for $M$ is obtained for the maximum value of $\alpha$, while for $\psi=c$ the maximum for $M$ is obtained for the minimum value of $\alpha$. Finally, the maximum is obtained exactly for $\alpha=\bar{\alpha}$.

For the optimization of the cost functionals $J_{1}(T)$ and $J_{2}(T)$, we make use of the following theorem.

THEOREM 3.2. Consider a junction $J$ with $n=1$ incoming road and $m=2$ outgoing roads. For the flux function (2.2) and $T$ sufficiently large, the cost functionals $J_{1}(T)$ and $J_{2}(T)$ are optimized for $\alpha=\frac{1}{2}$, with the exception of the following cases where the optimal control does not exist but the optimal value is approximated for $\varepsilon$ small and positive:

1. if $\gamma_{b}^{\max }<\gamma_{a}^{\max } \leq \gamma_{c}^{\max }$ and $\gamma_{b}^{\max } \leq \frac{\gamma_{a}^{\max }}{2}$, one can choose $\alpha=\frac{\gamma_{b}^{\max }}{\gamma_{a}^{\max }}-\varepsilon$;

2. if $\gamma_{c}^{\max }<\gamma_{a}^{\max } \leq \gamma_{b}^{\max }$ and $\gamma_{c}^{\max } \leq \frac{\gamma_{a}^{\max }}{2}$, one can choose $\alpha=1-\frac{\gamma_{c}^{\max }}{\gamma_{a}^{\max }}+\varepsilon$;

3. if $\frac{\gamma_{b}^{\max }}{\gamma_{a}^{\max }}<\frac{\gamma_{b}^{\max }}{\gamma_{b}^{\max }+\gamma_{c}^{\max }}<1-\frac{\gamma_{c}^{\max }}{\gamma_{a}^{\max }}$, we have two cases: if $\gamma_{b}^{\max }<\gamma_{c}^{\max }<\gamma_{a}^{\max }$, one can choose $\alpha=\frac{\gamma_{b}^{\max }}{\gamma_{b}^{\max }+\gamma_{c}^{\max }}-\varepsilon$; otherwise, if $\gamma_{c}^{\max }<\gamma_{b}^{\max }<\gamma_{a}^{\max }$, one can choose $\alpha=\frac{\gamma_{b}^{\max }}{\gamma_{b}^{\max }+\gamma_{c}^{\max }}+\varepsilon$; 
4. if $\gamma_{b}^{\max } \leq \gamma_{c}^{\max }<\gamma_{a}^{\max }$, and $\alpha_{1}<\frac{\gamma_{b}^{\max }}{\gamma_{b}^{\max }+\gamma_{c}^{\max }}<\alpha_{2}$, where $\alpha_{1}=1-\frac{\gamma_{c}^{\max }}{\gamma_{a}^{\max }}$ and $\alpha_{2}=$ $\frac{\gamma_{b}^{\max }}{\gamma_{a}^{\max }}$, we have to distinguish between two cases:

- if $\alpha_{2}=\frac{1}{2}$, one can choose $\alpha=\frac{1}{2}-\varepsilon$;

- if $\alpha_{1}<\alpha_{2} \leq \frac{1}{2}$, one can choose $\alpha=\alpha_{2}-\varepsilon$.

REMARK 3.3. Theorem 3.2 is proved by estimating the flux values $\widehat{\gamma}_{a}$ and the derivatives of $J_{i}$ with respect to $\widehat{\gamma}_{a}$. Then, the results also hold for the case of fluxes $f_{\varphi}=a_{\varphi} \rho(1-\rho)$ on a $\operatorname{road} I_{\varphi}$, with $a_{\varphi}>0, \varphi \in\{a, b, c\}$.

Proof. For simplicity, from now on we drop the dependence on $T$ in $J_{1}$ and $J_{2}$.

We distinguish various cases.

First case

We assume that (Figure 3.1)

$$
\gamma_{a}^{\max }<\gamma_{c}^{\max }<\gamma_{b}^{\max }
$$

or

$$
\gamma_{a}^{\max }<\gamma_{b}^{\max }<\gamma_{c}^{\max }
$$
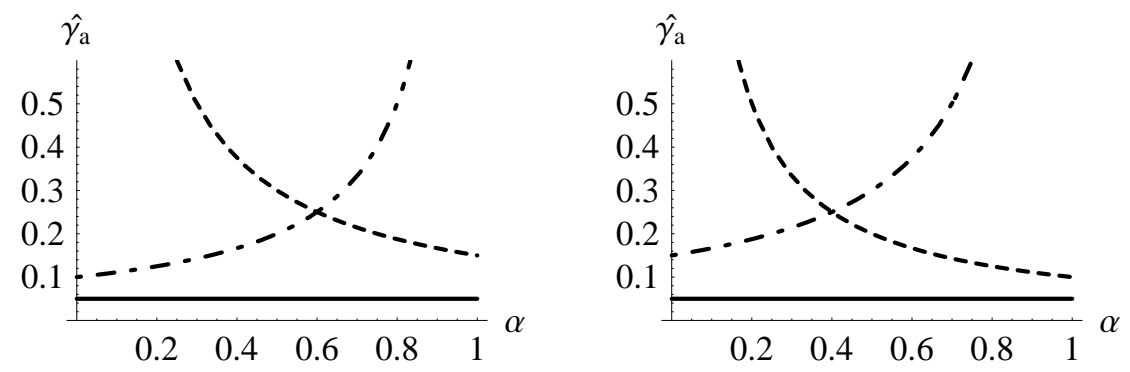

FIG. 3.1. Case $\gamma_{a}^{\max }<\gamma_{c}^{\max }<\gamma_{b}^{\max }$ (left). We considered the case $\gamma_{a}^{\max }=0.05, \gamma_{b}^{\max }=0.15$, and $\gamma_{c}^{\max }=0.1$. Continuous line: $\gamma_{a}^{\max }$; dashed line: $\frac{\gamma_{b}^{\max }}{\alpha}$; dot dashed line: $\frac{\gamma_{c}^{\max }}{1-\alpha}$. Case $\gamma_{a}^{\max }<$ $\gamma_{b}^{\max }<\gamma_{c}^{\max }$ (right). We considered the case $\gamma_{a}^{\max }=0.05, \gamma_{b}^{\max }=0.1$, and $\gamma_{c}^{\max }=0.15$. Continuous line: $\gamma_{a}^{\max }$; dashed line: $\frac{\gamma_{b}^{\max }}{\alpha}$; dot dashed line: $\frac{\gamma_{c}^{\max }}{1-\alpha}$.

If (3.5) or (3.6) hold, then

$$
\widehat{\gamma}_{a}=\gamma_{a}^{\max }
$$

As $s_{b}=s_{c}=-1$, we have to maximize

$$
\widehat{J_{1}}=\sqrt{1-4 \alpha \gamma_{a}^{\max }}+\sqrt{1-4(1-\alpha) \gamma_{a}^{\max }}
$$

and minimize

$$
\widehat{J}_{2}=\frac{1}{1+\sqrt{1-4 \alpha \gamma_{a}^{\max }}}+\frac{1}{1+\sqrt{1-4(1-\alpha) \gamma_{a}^{\max }}}
$$


The expressions $\widehat{J}_{1}$ and $\widehat{J}_{2}$ are defined for $1-\frac{1}{4 \gamma_{a}^{\max }} \leq \alpha \leq \frac{1}{4 \gamma_{a}^{\max }}$, which is to say that $\widehat{J}_{1}$ and $\widehat{J}_{2}$ are completely defined for $\left.\alpha \in\right] 0,1\left[\right.$. In fact, $\gamma_{a}^{\max } \in[0,0.25]$. We notice that

$$
\begin{gathered}
\frac{\partial \widehat{J}_{1}}{\partial \alpha}=-\frac{2 \gamma_{a}^{\max }}{\sqrt{1-4 \alpha \gamma_{a}^{\max }}}+\frac{2 \gamma_{a}^{\max }}{\sqrt{1-4(1-\alpha) \gamma_{a}^{\max }}} \\
\frac{\partial \widehat{J}_{2}}{\partial \alpha}=\Phi(\alpha)-\Phi(1-\alpha)
\end{gathered}
$$

where

$$
\Phi(\alpha)=\frac{2 \gamma_{a}^{\max }}{\left[1+\sqrt{1-4 \alpha \gamma_{a}^{\max }}\right]^{2} \sqrt{1-4 \alpha \gamma_{a}^{\max }}} .
$$

Since

$$
\begin{gathered}
\frac{\partial \widehat{J}_{1}}{\partial \alpha} \geq 0 \Leftrightarrow \alpha \leq \frac{1}{2}, \\
\frac{\partial \widehat{J}_{2}}{\partial \alpha} \geq 0 \Leftrightarrow \alpha \geq \frac{1}{2},
\end{gathered}
$$

we can conclude that the maximization of $J_{1}$ and the minimization of $J_{2}$ occur for $\alpha=\frac{1}{2}$. Notice that this last result is achieved also when $\gamma_{a}^{\max }=\gamma_{b}^{\max }=\gamma_{c}^{\max }$.

Second case

We assume that (Figure 3.2, left)

$$
\gamma_{b}^{\max }<\gamma_{a}^{\max }<\gamma_{c}^{\max } .
$$
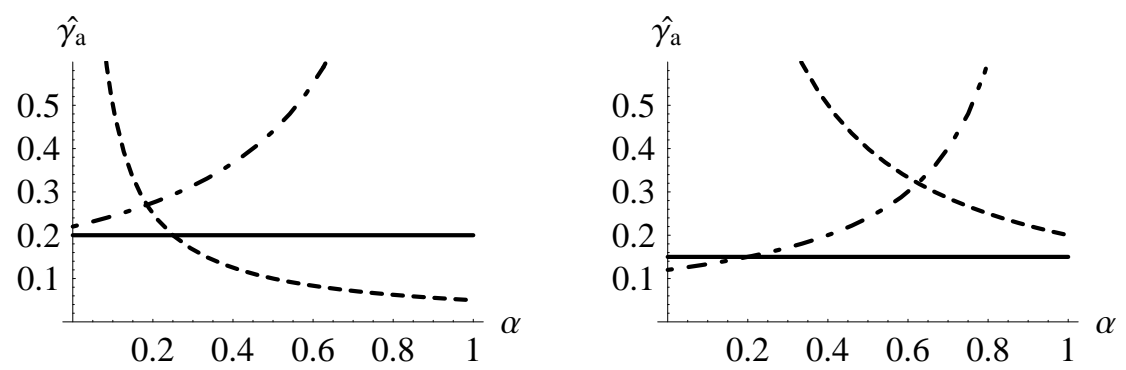

FIG. 3.2. Case $\gamma_{b}^{\max }<\gamma_{a}^{\max }<\gamma_{c}^{\max }$ (left). We considered the case $\gamma_{a}^{\max }=0.2, \gamma_{b}^{\max }=0.05$, and $\gamma_{c}^{\max }=0.22$. Continuous line: $\gamma_{a}^{\max }$; dashed line: $\frac{\gamma_{b}^{\max }}{\alpha}$; dot dashed line: $\frac{\gamma_{c}^{\max }}{1-\alpha}$. Case $\gamma_{c}^{\max }<$ $\gamma_{a}^{\max }<\gamma_{b}^{\max }$ (right). We considered the case $\gamma_{a}^{\max }=0.15, \gamma_{b}^{\max }=0.2$, and $\gamma_{c}^{\max }=0.12$. Continuous line: $\gamma_{a}^{\max }$; dashed line: $\frac{\gamma_{b}^{\max }}{\alpha}$; dot dashed line: $\frac{\gamma_{c}^{\max }}{1-\alpha}$.

If (3.9) holds, then

$$
\widehat{\gamma}_{a}= \begin{cases}\gamma_{a}^{\max }, & \text { if } 0<\alpha \leq \bar{\alpha} \\ \frac{\gamma_{b}^{\max }}{\alpha}, & \text { if } \bar{\alpha}<\alpha<1\end{cases}
$$


where

$$
\bar{\alpha}=\frac{\gamma_{b}^{\max }}{\gamma_{a}^{\max }}
$$

Then, as for $0<\alpha \leq \bar{\alpha}, s_{b}=s_{c}=-1$, and for $\bar{\alpha}<\alpha<1, s_{a}=+1, s_{c}=-1$, we have to maximize

$$
\widehat{J}_{1}= \begin{cases}+\sqrt{1-4 \alpha \gamma_{a}^{\max }}+\sqrt{1-4(1-\alpha) \gamma_{a}^{\max }}, & \text { if } 0<\alpha \leq \bar{\alpha}, \\ -\sqrt{1-4 \frac{\gamma_{b}^{\max }}{\alpha}}+\sqrt{1-4 \frac{1-\alpha}{\alpha} \gamma_{b}^{\max }}, & \text { if } \bar{\alpha}<\alpha<1\end{cases}
$$

and to minimize

$$
\widehat{J}_{2}= \begin{cases}\frac{1}{1+\sqrt{1-4 \alpha \gamma_{a}^{\max }}}+\frac{1}{1+\sqrt{1-4(1-\alpha) \gamma_{a}^{\max }}}, & \text { if } 0<\alpha \leq \bar{\alpha}, \\ \frac{1}{1-\sqrt{1-4 \frac{\gamma_{b}^{\max }}{\alpha}}}+\frac{1}{1+\sqrt{1-4 \frac{1-\alpha}{\alpha} \gamma_{b}^{\max }}} & \text { if } \bar{\alpha}<\alpha<1 .\end{cases}
$$

Let us examine the situation for $0<\alpha \leq \bar{\alpha}$. In this case, $\frac{\partial \widehat{J}_{1}}{\partial \alpha}$ and $\frac{\partial \widehat{J}_{2}}{\partial \alpha}$ are, respectively, the expressions (3.7) and (3.8), and the analysis is made as before. If $\bar{\alpha}<\alpha<1$,

$$
\begin{gathered}
\frac{\partial \widehat{J}_{1}}{\partial \alpha}=2 \frac{\gamma_{b}^{\max }}{\alpha^{2}}\left[-\frac{1}{\sqrt{1-4 \frac{\gamma_{b}^{\max }}{\alpha}}}+\frac{1}{\sqrt{1-4 \frac{1-\alpha}{\alpha} \gamma_{b}^{\max }}}\right], \\
\frac{\partial \widehat{J}_{2}}{\partial \alpha}=m(\alpha)+n(\alpha),
\end{gathered}
$$

where

$$
\begin{gathered}
m(\alpha)=\frac{2 \gamma_{b}^{\max }}{\alpha^{2}} \frac{1}{\left(-1+\sqrt{1-4 \frac{\gamma_{b}^{\max }}{\alpha}}\right)^{2} \sqrt{1-4 \frac{\gamma_{b}^{\max }}{\alpha}}}, \\
n(\alpha)=-\frac{2 \gamma_{b}^{\max }}{\alpha^{2}} \frac{1}{\left(1+\sqrt{1-4 \frac{1-\alpha}{\alpha} \gamma_{b}^{\max }}\right)^{2} \sqrt{1-4 \frac{1-\alpha}{\alpha} \gamma_{b}^{\max }}} .
\end{gathered}
$$

If $\bar{\alpha}<\alpha<1$, from the analysis of (3.10) and (3.11) $\widehat{J}_{1}$ and $\widehat{J_{2}}$ are, respectively, a decreasing and an increasing function $\forall \alpha \in\left[4 \gamma_{b}^{\max }, 1[\right.$.

We can collect the following results for the cost functional $J_{1}$

- if $\bar{\alpha} \leq \frac{1}{2}$, the optimal value for $J_{1}$ does not exist. One can choose $\alpha=\bar{\alpha}-\varepsilon$;

- if $\bar{\alpha}>\frac{1}{2}, J_{1}$ is optimized for $\alpha=\frac{1}{2}$. 
For $J_{2}$, we have the same results obtained for $J_{1}$.

Notice that, if $\gamma_{a}^{\max }=\gamma_{b}^{\max }$, or $\gamma_{a}^{\max }=\gamma_{b}^{\max }=\gamma_{c}^{\max }$, the optimal values for $J_{1}$ and $J_{2}$ are obtained for $\alpha=\frac{1}{2}$. If $\gamma_{a}^{\max }=\gamma_{c}^{\max }$, the analysis of the second case is unchanged.

We similarly treat the symmetric case (see Figure 3.2, right), where we assume that

$$
\gamma_{c}^{\max }<\gamma_{a}^{\max }<\gamma_{b}^{\max }
$$

If (3.12) holds, then

$$
\widehat{\gamma}_{a}= \begin{cases}\frac{\gamma_{c}^{\max }}{1-\alpha}, & \text { if } 0<\alpha \leq \bar{\alpha} \\ \gamma_{a}^{\max }, & \text { if } \bar{\alpha}<\alpha<1\end{cases}
$$

where

$$
\bar{\alpha}=1-\frac{\gamma_{c}^{\max }}{\gamma_{a}^{\max }} .
$$

Then, as for $0<\alpha \leq \bar{\alpha}, s_{a}=+1, s_{b}=-1$, for $\bar{\alpha}<\alpha<1, s_{b}=s_{c}=-1$, we have to maximize

$$
\widehat{J}_{1}= \begin{cases}-\sqrt{1-4 \frac{\gamma_{c}^{\max }}{1-\alpha}}+\sqrt{1-4 \frac{\alpha}{1-\alpha} \gamma_{c}^{\max }}, & \text { if } 0<\alpha \leq \bar{\alpha}, \\ +\sqrt{1-4 \alpha \gamma_{a}^{\max }}+\sqrt{1-4(1-\alpha) \gamma_{a}^{\max }}, & \text { if } \bar{\alpha}<\alpha<1,\end{cases}
$$

and minimize

$$
\widehat{J}_{2}= \begin{cases}\frac{1}{1-\sqrt{1-4 \frac{\gamma_{c}^{\max }}{1-\alpha}}}+\frac{1}{1+\sqrt{1-4 \frac{\alpha}{1-\alpha} \gamma_{c}^{\max }}}, & \text { if } 0<\alpha \leq \bar{\alpha}, \\ \frac{1}{1+\sqrt{1-4 \alpha \gamma_{a}^{\max }}}+\frac{1}{1+\sqrt{1-4(1-\alpha) \gamma_{a}^{\max }}}, & \text { if } \bar{\alpha}<\alpha<1 .\end{cases}
$$

If $0<\alpha \leq \bar{\alpha}$,

$$
\begin{gathered}
\frac{\partial \widehat{J}_{1}}{\partial \alpha}=2 \frac{\gamma_{c}^{\max }}{(1-\alpha)^{2}}\left[\frac{1}{\sqrt{1-4 \frac{\gamma_{c}^{\max }}{1-\alpha}}}-\frac{1}{\sqrt{1-4 \frac{\alpha}{1-\alpha} \gamma_{c}^{\max }}}\right] \\
\frac{\partial \widehat{J}_{2}}{\partial \alpha}=r(\alpha)+s(\alpha)
\end{gathered}
$$

where

$$
\begin{gathered}
r(\alpha)=-\frac{2 \gamma_{c}^{\max }}{(1-\alpha)^{2}} \frac{1}{\left(-1+\sqrt{1-4 \frac{\gamma_{c}^{\max }}{1-\alpha}}\right)^{2} \sqrt{1-4 \frac{\gamma_{c}^{\max } \frac{\alpha}{1-\alpha}}{2}}} \\
s(\alpha)=\frac{2 \gamma_{c}^{\max }}{(1-\alpha)^{2}} \frac{1}{\left(1+\sqrt{1-4 \frac{\alpha}{1-\alpha} \gamma_{c}^{\max }}\right)^{2} \sqrt{1-4 \frac{\alpha}{1-\alpha} \gamma_{c}^{\max }}} .
\end{gathered}
$$


If $0<\alpha<\bar{\alpha}$, from the analysis of (3.13) and (3.14) we get that $\widehat{J}_{1}$ and $\widehat{J}_{2}$ are, respectively, an increasing and a decreasing function $\forall \alpha \in\left[0,1-4 \gamma_{c}^{\max }\right]$.

If $\bar{\alpha}<\alpha<1$, from the analysis of (3.10) and (3.11) $\widehat{J}_{1}$ and $\widehat{J}_{2}$ are, respectively, a decreasing and an increasing function $\forall \alpha \in\left[4 \gamma_{b}^{\max }, 1\right.$ [. We can collect the following information for the cost functional $J_{1}$

- if $\bar{\alpha}<\frac{1}{2}, J_{1}$ is optimized for $\alpha=\frac{1}{2}$;

- if $\bar{\alpha} \geq \frac{1}{2}$, the optimal value for $J_{1}$ does not exist. One can choose $\alpha=\bar{\alpha}+\varepsilon$.

For $J_{2}$, we have the same results obtained for $\alpha$ of $J_{1}$.

Notice that if $\gamma_{a}^{\max }=\gamma_{b}^{\max }$, the analysis of this case is unchanged. If $\gamma_{a}^{\max }=\gamma_{c}^{\max }$, or $\gamma_{a}^{\max }=\gamma_{b}^{\max }=\gamma_{c}^{\max }$, the optimal value of $\alpha$ for $J_{1}$ and $J_{2}$ is $\alpha=\frac{1}{2}$.

Third case

We assume that (Figure 3.3)

$$
\gamma_{b}^{\max }<\gamma_{c}^{\max }<\gamma_{a}^{\max }
$$

or

$$
\gamma_{c}^{\max }<\gamma_{b}^{\max }<\gamma_{a}^{\max }
$$
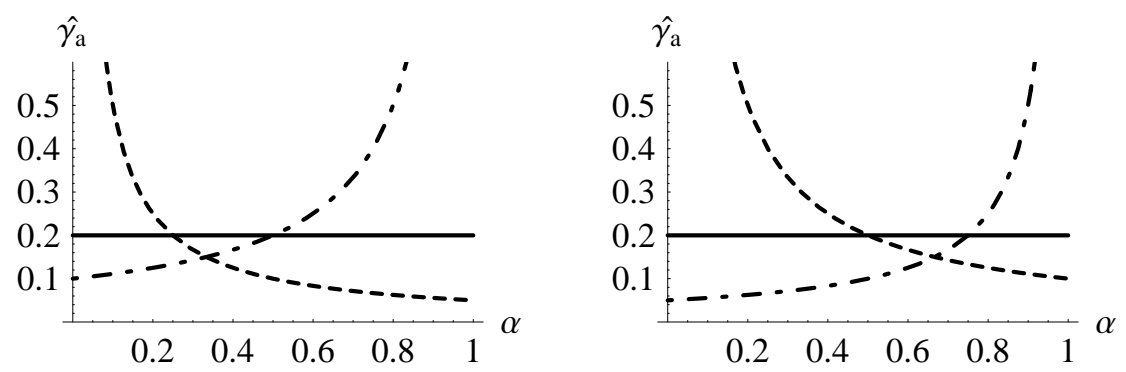

FIG. 3.3. Case $\gamma_{b}^{\max }<\gamma_{c}^{\max }<\gamma_{a}^{\max }$ (left). We considered the case $\gamma_{a}^{\max }=0.2, \gamma_{b}^{\max }=0.05$, and $\gamma_{c}^{\max }=0.1$. Continuous line: $\gamma_{a}^{\max } ;$ dashed line: $\frac{\gamma_{b}^{\max }}{\alpha}$; dot dashed line: $\frac{\gamma_{c}^{\max }}{1-\alpha}$. Case $\gamma_{c}^{\max }<\gamma_{b}^{\max }<$ $\gamma_{a}^{\max }$ (right). We considered the case $\gamma_{a}^{\max }=0.2, \gamma_{b}^{\max }=0.1$, and $\gamma_{c}^{\max }=0.05$. Continuous line: $\gamma_{a}^{\max } ;$ dashed line: $\frac{\gamma_{b}^{\max }}{\alpha}$; dot dashed line: $\frac{\gamma_{c}^{\max }}{1-\alpha}$.

If (3.15) or (3.16) hold, then

$$
\widehat{\gamma}_{a}= \begin{cases}\frac{\gamma_{c}^{\max }}{1_{\max }-\alpha}, & \text { if } 0<\alpha \leq \bar{\alpha}, \\ \frac{\gamma_{b}^{\max }}{\alpha}, & \text { if } \bar{\alpha}<\alpha<1,\end{cases}
$$

where

$$
\frac{\gamma_{b}^{\max }}{\gamma_{a}^{\max }}<\bar{\alpha}=\frac{\gamma_{b}^{\max }}{\gamma_{b}^{\max }+\gamma_{c}^{\max }}<1-\frac{\gamma_{c}^{\max }}{\gamma_{a}^{\max }}
$$


Then, as for $0<\alpha \leq \bar{\alpha}, s_{a}=+1, s_{b}=-1$, while, for $\bar{\alpha}<\alpha<1, s_{a}=+1, s_{c}=-1$, we have to maximize

$$
\widehat{J}_{1}= \begin{cases}-\sqrt{1-4 \frac{\gamma_{c}^{\max }}{1-\alpha}}+\sqrt{1-4 \frac{\alpha}{1-\alpha} \gamma_{c}^{\max }}, & \text { if } 0<\alpha \leq \bar{\alpha}, \\ -\sqrt{1-4 \frac{\gamma_{b}^{\max }}{\alpha}}+\sqrt{1-4 \frac{1-\alpha}{\alpha} \gamma_{b}^{\max }}, & \text { if } \bar{\alpha}<\alpha<1,\end{cases}
$$

and to minimize

$$
\widehat{J}_{2}= \begin{cases}\frac{1}{1-\sqrt{1-4 \frac{\gamma_{c}^{\max }}{1-\alpha}}}+\frac{1}{1+\sqrt{1-4 \frac{\alpha}{1-\alpha} \gamma_{c}^{\max }}}, & \text { if } 0<\alpha \leq \bar{\alpha}, \\ \frac{1}{1-\sqrt{1-4 \frac{\gamma_{b}^{\max }}{\alpha}}}+\frac{1}{1+\sqrt{1-4 \frac{1-\alpha}{\alpha} \gamma_{b}^{\max }}}, & \text { if } \bar{\alpha}<\alpha<1 .\end{cases}
$$

Remembering the results seen before, we can collect the following information for the cost functional:

- if (3.15) holds, the optimal value for $J_{1}$ does not exist. One can choose $\alpha=\bar{\alpha}-\varepsilon$;

- if (3.16) holds, the optimal value for $J_{1}$ does not exist. One can choose $\alpha=\bar{\alpha}+\varepsilon$.

For $J_{2}$, we have the same results obtained for $J_{1}$.

Notice that, if (3.15) holds, and if $\gamma_{a}^{\max }=\gamma_{c}^{\max }$, or $\gamma_{b}^{\max }=\gamma_{c}^{\max }$, we deal with the second case. If (3.16) holds, and if $\gamma_{a}^{\max }=\gamma_{b}^{\max }$, we deal with the second case. If (3.16) holds, and if $\gamma_{b}^{\max }=\gamma_{c}^{\max }$, or if $\gamma_{a}^{\max }=\gamma_{b}^{\max }=\gamma_{c}^{\max }$, we refer to the first case.

Fourth case

We assume that (see Figure 3.4)

$$
\gamma_{b}^{\max }<\gamma_{c}^{\max }<\gamma_{a}^{\max }
$$

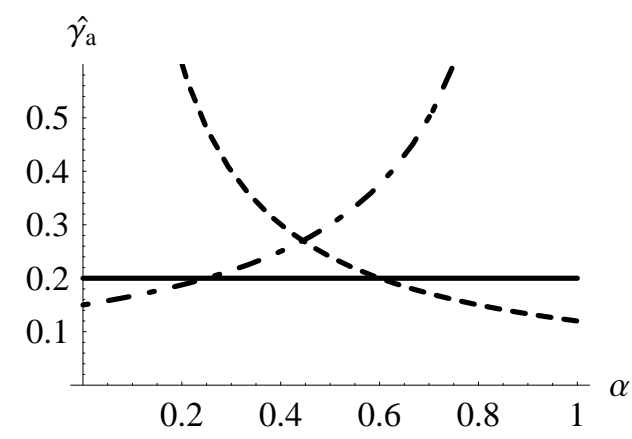

FIG. 3.4. Case $\gamma_{b}^{\max }<\gamma_{c}^{\max }<\gamma_{a}^{\max }$. We considered the case $\gamma_{a}^{\max }=0.2, \gamma_{b}^{\max }=0.12$, and $\gamma_{c}^{\max }=0.15$. Continuous line: $\gamma_{a}^{\max } ;$ dashed line: $\frac{\gamma_{b}^{\max }}{\alpha} ;$ dot dashed line: $\frac{\gamma_{c}^{\max }}{1-\alpha}$. 
If (3.17) holds, then

$$
\widehat{\gamma}_{a}=\left\{\begin{array}{cc}
\frac{\gamma_{c}^{\max }}{1-\alpha}, & \text { if } 0<\alpha \leq \alpha_{1}, \\
\gamma_{a}^{\max }, & \text { if } \alpha_{1}<\alpha<\alpha_{2}, \\
\frac{\gamma_{b}^{\max }}{\alpha}, & \text { if } \alpha_{2} \leq \alpha<1,
\end{array}\right.
$$

where

$$
\alpha_{1}=1-\frac{\gamma_{c}^{\max }}{\gamma_{a}^{\max }}<\frac{\gamma_{b}^{\max }}{\gamma_{b}^{\max }+\gamma_{c}^{\max }}
$$

and

$$
\alpha_{2}=\frac{\gamma_{b}^{\max }}{\gamma_{a}^{\max }}>\frac{\gamma_{b}^{\max }}{\gamma_{b}^{\max }+\gamma_{c}^{\max }} .
$$

For $0<\alpha \leq \alpha_{1}$, we have that $s_{a}=+1$, and $s_{b}=-1$, while for $\alpha_{1}<\alpha<\alpha_{2}$, we have that $s_{b}=s_{c}=-1$. Finally, for $\alpha_{2} \leq \alpha<1$, we have that $s_{a}=+1$, and $s_{c}=-1$. Then, we have to maximize

$$
\widehat{J}_{1}=\left\{\begin{array}{cc}
-\sqrt{1-4 \frac{\gamma_{c}^{\max }}{1-\alpha}}+\sqrt{1-4 \frac{\alpha}{1-\alpha} \gamma_{c}^{\max }}, & \text { if } 0<\alpha \leq \alpha_{1}, \\
+\sqrt{1-4 \alpha \gamma_{a}^{\max }}+\sqrt{1-4(1-\alpha) \gamma_{a}^{\max }}, & \text { if } \alpha_{1}<\alpha<\alpha_{2}, \\
-\sqrt{1-4 \frac{\gamma_{b}^{\max }}{\alpha}}+\sqrt{1-4 \frac{1-\alpha}{\alpha} \gamma_{b}^{\max }}, & \text { if } \alpha_{2} \leq \alpha<1,
\end{array}\right.
$$

and minimize

$$
\widehat{J}_{2}= \begin{cases}\frac{1}{1-\sqrt{1-4 \frac{\gamma_{c}^{\max }}{1-\alpha}}}+\frac{1}{1+\sqrt{1-4 \frac{\alpha}{1-\alpha} \gamma_{c}^{\max }},} & \text { if } 0<\alpha \leq \alpha_{1}, \\ \frac{1}{1+\sqrt{1-4 \alpha \gamma_{a}^{\max }}}+\frac{1}{1+\sqrt{1-4(1-\alpha) \gamma_{a}^{\max }}}, & \text { if } \alpha_{1}<\alpha<\alpha_{2}, \\ \frac{1}{1-\sqrt{1-4 \frac{\gamma_{b}^{\max }}{\alpha}}}+\frac{1}{1+\sqrt{1-4 \frac{1-\alpha}{\alpha} \gamma_{b}^{\max }}}, & \text { if } \alpha_{2} \leq \alpha<1 .\end{cases}
$$

Remembering the results seen before, we can collect the following information for the cost functional $J_{1}$

- if $\alpha_{1}<\frac{1}{2}<\alpha_{2}, J_{1}$ is optimized for $\alpha=\frac{1}{2}$. If $\alpha_{2}=\frac{1}{2}$, the optimal for $J_{1}$ does not exist. One can choose $\alpha=\frac{1}{2}-\varepsilon$;

- if $\alpha_{1}<\alpha_{2} \leq \frac{1}{2}$, the optimal for $J_{1}$ does not exist. One can choose $\alpha=\alpha_{2}-\varepsilon$.

For $J_{2}$, we have the same results obtained for $J_{1}$.

Notice that, if $\gamma_{b}^{\max }=\gamma_{c}^{\max }$, the analysis is unchanged. If $\gamma_{a}^{\max }=\gamma_{c}^{\max }$, we refer to the analysis of the second case; if $\gamma_{a}^{\max }=\gamma_{b}^{\max }=\gamma_{c}^{\max }$, we refer to the analysis of the first case.

Fifth case

Assume that $\gamma_{b}^{\max }=0$, or $\gamma_{c}^{\max }=0$, or $\gamma_{b}^{\max }=\gamma_{c}^{\max }=0$. In this case, $\widehat{\gamma}_{a} \equiv 0 \forall \alpha \in$ ]0,1[. The optimal case is achieved for an arbitrary value of $\alpha$. Thus, we can choose $\alpha=\frac{1}{2}$. 


\section{Simulation of traffic}

This section is devoted to the presentation of simulations for a road network, that consists of six junctions of $1 \times 2$ and $2 \times 2$ type (two incoming roads and two outgoing roads). Such a network can be considered as a good example of real road networks and it is useful for understanding the traffic conditions for various values of the distribution coefficients, whose optimization was presented in the previous section for junctions of $1 \times 2$ type. In particular, for every junction of $2 \times 2$ type, the distribution matrix $A$ assumes the form:

$$
A=\left(\begin{array}{ll}
0.5 & 0.5 \\
0.5 & 0.5
\end{array}\right)
$$

Hence, no control is considered for such junctions.

The performance of the network is evaluated through the cost functionals $J_{1}$ and $J_{2}$. Their evolution is strongly influenced by the distribution coefficients, which is to say that traffic conditions are strictly connected to the behavior of drivers, a factor that, from one side, is easily deducible and, from the other, is confirmed by simulations.

We study the network of Figure 4.1, for which we have the following features:

- external roads: $a_{1}, a_{3}, c_{2}, e_{2}, f_{1}$, and $f_{2}$;

- inner roads: $a_{2}, b_{1}, b_{2}, c_{1}, d_{1}, d_{2}$, and $e_{1}$;

- four junctions of $1 \times 2$ type: $1,2,3,5$;

- two junctions of $2 \times 2$ type: 4,6 .

For external roads of the presented network, boundary conditions are needed.

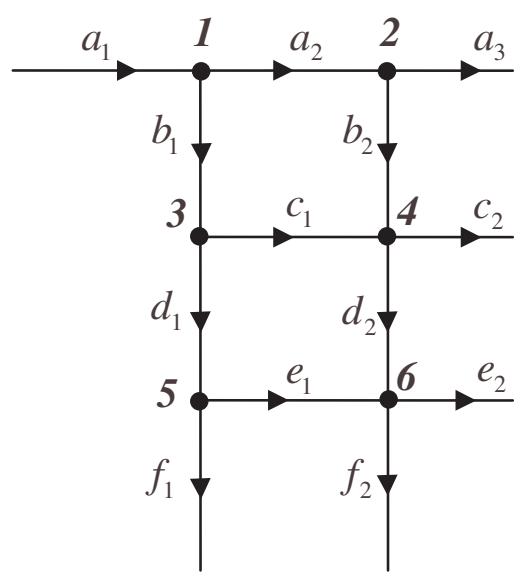

FIG. 4.1. Topology of the network.

4.1. Simulation Characteristics. In this section, we examine some simulation results for different choices of the distribution parameters. The approximation of the conservation laws that describe the evolution of densities for each road of the network is made by the numerical scheme of Godunov ([11]), with spatial step 
$\Delta x=0.01$. The time step is determined by the CFL condition ([10]), equal to 0.5 . The flux function chosen for simulation is given by (2.2).

The network of Figure 4.1 is simulated with the following conditions: initial data equal to 0.3 for all roads at the starting instant of simulation $(t=0)$; boundary data of Dirichlet type, equal to 0.45 for road $a_{1}$, while for roads $a_{3}, c_{2}, f_{1}$, and $f_{2}$, we choose a Dirichlet boundary data equal to 0.9 ; the time interval of simulation is $[0, T]$, where $T=30 \mathrm{~min}$.

Three different choices of the distribution parameters are taken into account. In the optimal case, parameters, that locally optimize the asymptotic cost functionals through the analytical results obtained in the previous section, are used. In the fixed case, the distribution coefficient $\alpha$ for each junction is assumed to be the same. In the dynamic random case, the distribution coefficients change randomly at every step of the simulation process and independently for every junction. The last case permits to make some interesting discussions about the performances of the network.

\subsection{Simulation results.}

4.2.1. Fixed and optimal simulations. For the network of Figure 4.1, we can analyze traffic conditions for different values of $\alpha$. We refer to Figure 4.2 for $\alpha=0.2$, where the density $\rho(t, x)$ on roads $b_{1}$ (from 0 to 1 on the axis $x$ ), $d_{1}$ (from 1 to 2 on the axis $x$ ), and $f_{1}$ (from 2 to 3 on the axis $x$ ), is reported. In this case, high levels of density occur on these vertical roads, hence they tend to be more heavily congested than others. This can be seen in Figure 4.2 where, at $t=10$, the road $f_{1}$ is already congested with a density value almost equal to 0.9 . At $t=25$, the intense traffic on road $f_{1}$ propagates backward and influences roads $b_{1}$ and $d_{1}$. The traffic densities on other roads is very low.

When we deal with the optimal choice of the distribution coefficients, densities on roads $c_{2}, e_{2}, f_{1}$ and $f_{2}$ tend to increase. However, the optimal choice better redistributes traffic flows on the whole network, as we can see from Figure 4.3, that shows the car density $\rho(t, x)$ for roads $b_{1}, d_{1}$ and $f_{1}$.

Then, we compare three scenarios $(\alpha=0.2, \alpha=0.8$ and optimal $\alpha)$. We can conclude that a real decongestion effect is evident for optimal distribution coefficients (see Figure 4.4, that shows the cost functionals $J_{1}$ and $J_{2}$ ).

4.2.2. Dynamic random simulations and their interpretation. We now focus our attention on the dynamic random simulation. Such choice follows the behavior of the optimal one, as we can see from Figure 4.5. One could ask if an optimization is necessary, since random choices leads to similar functional values. The dynamic random simulation, in the reality of urban networks, implies that driver flow is very chaotic, since drivers' choices rapidly change during their own travel. Let us show this phenomenon considering the Stop and Go Waves functional ( $S G W$ ) (a systematic presentation is in [7] for the single road, and in [3] for road networks). The latter estimates the security of drivers travelling on the network, and it is defined as:

$$
S G W=\int_{0}^{T} \int_{\cup I_{i}}|D v(\rho)| d t d x .
$$

Figure 4.5 shows how $S G W$ indicates a great variation of velocity for the dynamic random choice, which implies a higher probability of car accidents. Note that the optimal case for $S G W$ is simulated according to the optimization algorithm for the cost functionals $J_{1}$ and $J_{2}$ (and not for $S G W$ itself). 


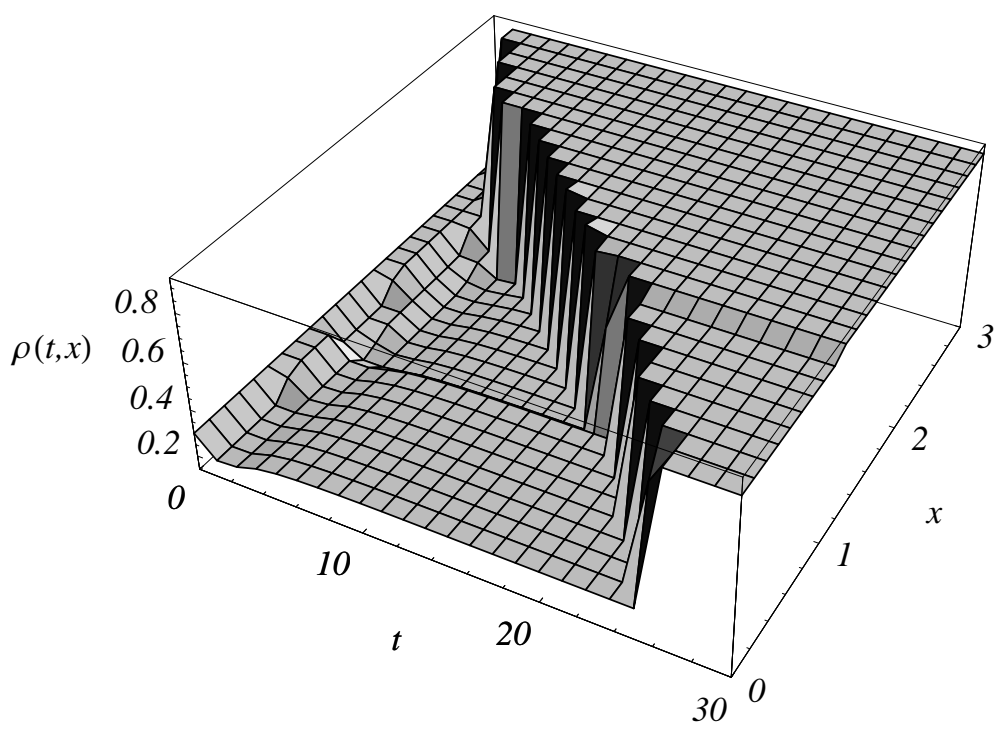

FIG. 4.2. Density $\rho(t, x)$ on roads $b_{1}$ (from 0 to 1 on the axis $x$ ), $d_{1}$ (from 1 to 2 on the axis $x$ ) and $f_{1}$ (from 2 to 3 on the axis $x$ ), for a choice of distribution coefficients all equal to 0.2 for $1 \times 2$ junctions.

From a statistical point of view, it is possible to understand why dynamic random simulations are very similar to the optimal case for functionals $J_{1}$ and $J_{2}$. From Theorem 3.2, the optimal choice for the distribution coefficient is almost always 0.5 , and this is the expected average value of random choices. Let us analyze this fact in more details.

Let $t \rightarrow \alpha(t)$ be the random process generated by the dynamic random simulation, whose statistical interpretation is the following: $P\{\alpha(t)=\alpha, 0 \leq \alpha \leq 1\}$ is the probability that, at the instant $t$, the distribution coefficient is equal to $\alpha$. For a fixed simulation time $\tau$, it is possible to define the temporal mean value of the process $\{\alpha(t), 0 \leq t \leq \tau\}$ as

$$
g(\tau, \alpha(t))=\frac{1}{\tau} \int_{0}^{\tau} \alpha(t) d t
$$

If the process $\{\alpha(t), 0 \leq t \leq \tau\}$ is ergodic (see [18]), then:

$$
\lim _{\tau \rightarrow \infty} g(\tau, \alpha(t))=E[\alpha(t)]
$$

where $E[\alpha(t)]$ represents the statistical mean value of the process $\{\alpha(t), 0 \leq t \leq \tau\}$. The quantity $E[\alpha(t)]$ is very simple to compute as, for a fixed instant of time $t$, $\{\alpha(t), 0 \leq t \leq \tau\}$ can be seen as a uniform random variable, $X_{t}$, in the real interval $[0,1]$. Hence,

$$
\lim _{\tau \rightarrow \infty} g(\tau, \alpha(t))=E[\alpha(t)]=E\left[X_{t}\right]=\frac{0+1}{2}=0.5
$$




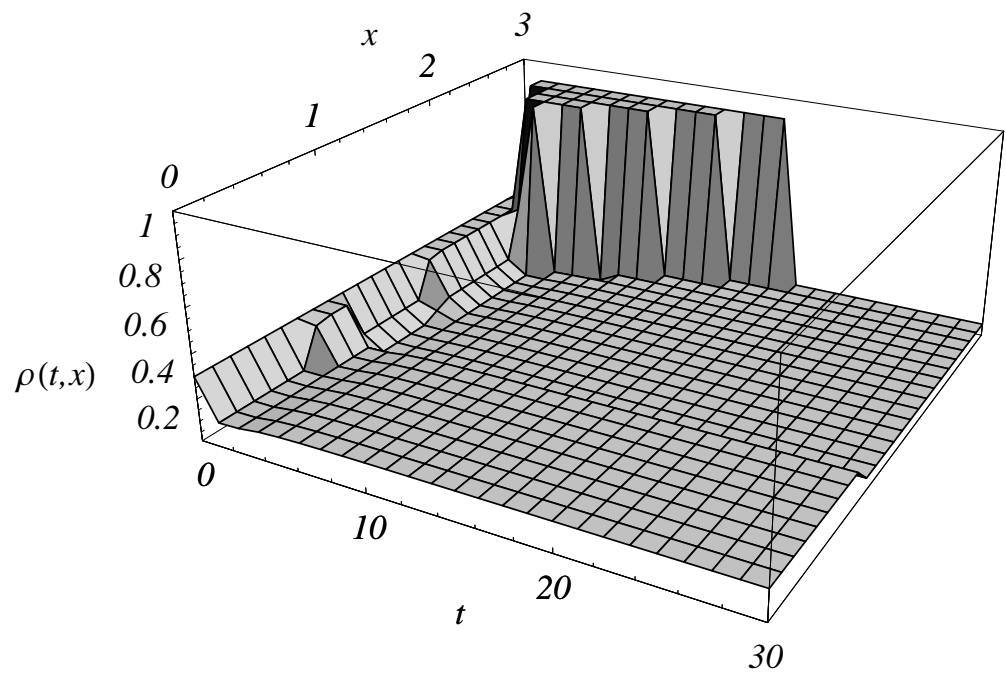

FIG. 4.3. Density $\rho(t, x)$ on roads $b_{1}$ (from 0 to 1 on the axis $x$ ), $d_{1}$ (from 1 to 2 on the axis $x$ ) and $f_{1}$ (from 2 to 3 on the axis $x$ ), for a choice of optimal distribution coefficients for $1 \times 2$ junctions.
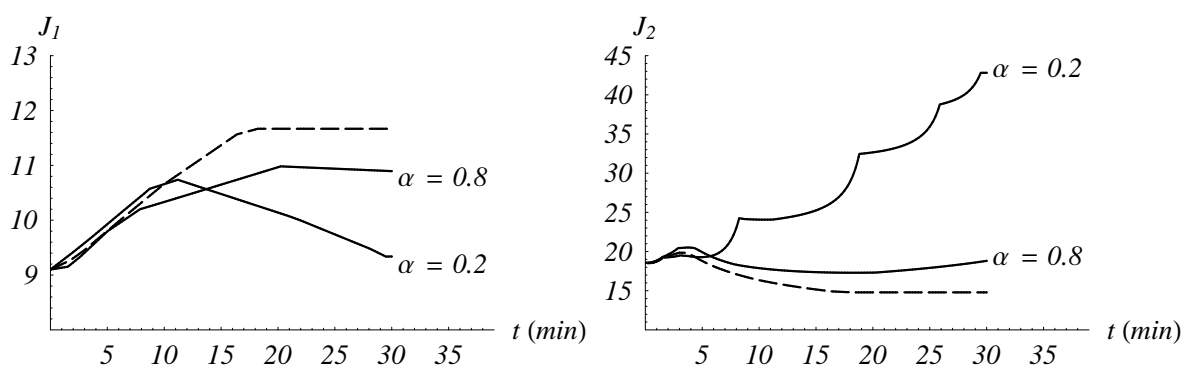

FIG. 4.4. $J_{1}$ (left) and $J_{2}$ (right). Solid lines: fixed cases for different values of the distribution coefficient; dashed line: optimal simulation.
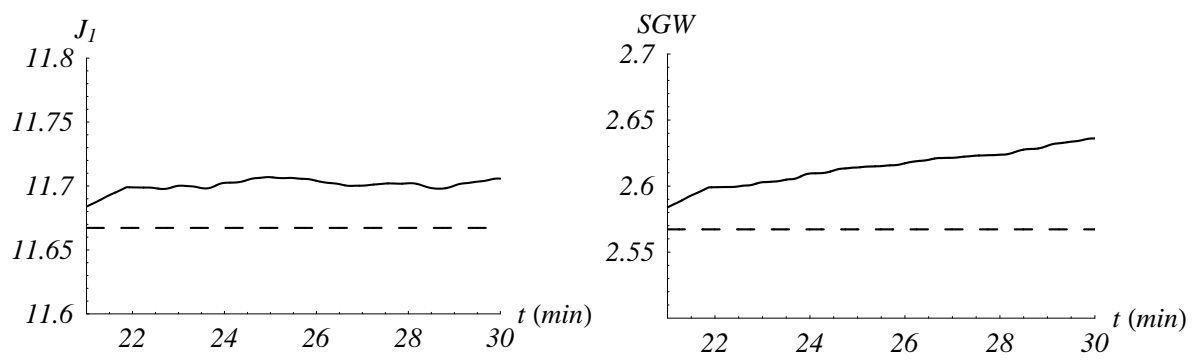

FIG. 4.5. Left: comparison among the dynamic random simulation and the optimal case for $J_{1}$. Right: behavior of $S G W$ in the optimal and in the dynamic random case; dashed line: optimal simulation. 
From a numerical point of view, we can verify that the random process is ergodic in such a way: let $[0, T]$ be the time interval that corresponds to the simulation. In order to define the discretization of the conservation laws for roads, it is necessary to define a spatial step $\Delta x$, from which the temporal step $\Delta t$ is easily obtained by the CFL condition (see [10]). Hence, the time interval $[0, T]$ is divided into $N$ subintervals, whose length is $\Delta t=t_{i}-t_{i-1} \forall i=1, \ldots, N$, with the assumption that $t_{0}=0, t_{N}=T$. In every instant of time $t_{i}$, a new parameter $0 \leq \alpha \leq 1$ for the junction is generated, hence this parameter is a uniform random variable $X_{t_{i}}$ in the real interval $[0,1]$. For every $t_{i}, i=0, \ldots, N$, we have that:

$$
\alpha\left(t_{i}\right)=X_{t_{i}} \sim U(0,1)
$$

Then, the continuous random process $\alpha(t)$ is numerically simulated as a discrete set of uniform random variables

$$
\chi=\left\{X_{t_{i}}\right\}_{i=0, . ., N},
$$

with cardinality $|\chi|=N+1$. Then the term (4.1) is approximated by

$$
\frac{1}{N+1} \sum_{i=0}^{N} X_{t_{i}} .
$$

Since $N$ is very large, according to the Strong Law of Large Numbers (4.3) represents a random variable whose mean value is

$$
E\left[\frac{1}{N+1} \sum_{i=0}^{N} X_{t_{i}}\right] \underset{N \rightarrow \infty}{\rightarrow} E\left[X_{t_{i}}\right]=0.5 .
$$

Thus, we have shown that the numerical implementation of $\alpha(t)$ is naturally ergodic. In Figure 4.6, a possible behavior for the random stochastic process $\alpha(t)$ and a picture of the temporal mean value of $\alpha(t), g(\tau, \alpha(t))$, are reported. We can see how the coefficient $\alpha$ is greatly variable in time and how the function $g(\tau, \alpha(t))$ converges to 0.5 when time increases.
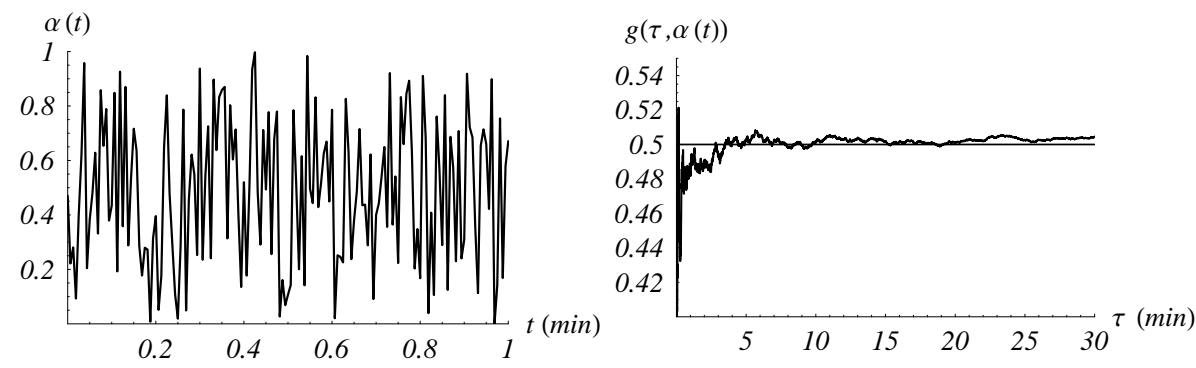

FIG. 4.6. Left: process $\alpha(t)$ generated during a simulation process. Right: temporal behavior of $g(\tau, \alpha(t))$.

\section{Conclusions}

In this paper, a traffic regulation technique for the optimization of car traffic flows in congested urban networks was considered. The model used for the network 
is of fluid dynamic type and the study of the performance was made through the use of three cost functionals, that give information about average velocity, average travelling time, and flux of cars on the network. Exact analytical results were given in a simple case, and then used in order to simulate more complex urban networks. It was shown that the optimal algorithm permits us to distribute traffic so as to avoid congestion in roads with high car densities, unlike fixed and dynamic random choices of the distribution parameters. The Stop and Go Waves functional and the study of the statistical behavior of dynamic random simulations allow us to establish that a dynamic random analysis has to be totally avoided for the car traffic regulation and decongestion.

\section{REFERENCES}

[1] A. Bressan, Hyperbolic Systems of Conservation Laws — The One-Dimensional Cauchy Problem, Oxford Univ. Press, 2000.

[2] G. Bretti, R. Natalini and B. Piccoli, Numerical approximations of a traffic flow model on networks, Networks and Heterogeneous Media, 1, 57-84, 2006.

[3] A. Cascone, C. D'Apice, B. Piccoli and L. Rarità, Optimization of traffic on road networks, Math. Models Meth. Appl. Sci., 17/10, 1587-1617, 2007.

[4] A. Cascone, A. Marigo, B. Piccoli and L. Rarità, Decentralized optimal routing for packets flow on data networks, preprint DIIMA.

[5] Y. Chitour and B. Piccoli, Traffic circles and timing of traffic lights for cars flow, Discrete and Continous Dynamical Systems - Series B, 5, 599-630, 2005.

[6] G. Coclite, M. Garavello and B. Piccoli, Traffic flow on road networks, SIAM J. Math. Anal., $36,1862-1886,2005$.

[7] R.M. Colombo and A. Groli, Minimising stop and go waves to optimise traffic flow, Applied Mathematics Letters, 17/6, 697-701, 2004.

[8] M. Garavello and B. Piccoli, Traffic Flow on Networks, Applied Math. Series, American Institute of Mathematical Sciences, 1, 2006.

[9] M. Garavello, R. Natalini, B. Piccoli and A. Terracina, Conservation laws with discontinuous flux, Networks and Heterogeneous Media, 2, 159-179, 2007.

[10] E. Godlewsky and P. Raviart, Numerical Approximation of Hyperbolic Systems of Conservation Laws, Springer Verlag, Heidelberg 1996.

[11] S.K. Godunov, A finite difference method for the numerical computation of discontinous solutions of the equations of fluid dynamics, Mat. Sb., 47, 271-290, 1959.

[12] M. Gugat, M. Herty, A. Klar and G. Leugering, Optimal control for traffic flow networks, Journal of Optimization Theory and Applications, 126, 589-616, 2005.

[13] D. Helbing, S. Lämmer and J.P. Lebacque, Self-organized control of irregular or perturbed network traffic, Optimal Control and Dynamic Games, C. Deissenberg and R.F. Hartl eds., Springer, Dordrecht, 239-274, 2005.

[14] M. Herty and A. Klar, Modelling, Simulation and optimization of traffic flow networks, SIAM J. Sci. Comp., 25, 1066-1087, 2003.

[15] H. Holden and N.H. Risebro, A mathematical model of traffic flow on a network of unidirectional roads, SIAM J. Math. Anal., 26, 999-1017, 1995.

[16] J.P. Lebacque, The Godunov scheme and what it means for first order traffic flow models, Proceedings of the Internaional Symposium on Transportation and Traffic Theory No13, Lyon , Pergamon Press, Oxford, 647-677, 1996.

[17] M.J. Lighthill and G.B. Whitham, On kinetic waves. II. theory of traffic flows on long crowded roads, Proc. Roy. Soc. London Ser. A, 229, 317-345, 1955.

[18] A. Papoulis and S. Unnikrishna Pillai, Probability, Random Variables and Stochastic Processes, Mc Graw Hill, 2002.

[19] P.I. Richards, Shock waves on the highway, Oper. Res., 4, 42-51, 1956. 